\title{
The inference of HIV-1 transmission direction between HIV-1 positive couples based on the sequences of HIV-1 quasi-species
}

Jianjun $\mathrm{Wu}^{1}$, Zhongwang Hu${ }^{2}$, Hui Yao ${ }^{2}$, Hai Wang ${ }^{2}$, Yanhua Lei², Ping Zhong ${ }^{3}$, Yi Feng ${ }^{4}$, Hui Xing ${ }^{4}$, Yuelan Shen ${ }^{1}$, Lin Jin ${ }^{1}$, Aiwen Liu', Yizu Qin ${ }^{1}$, Lifeng Miao ${ }^{1}$, Bin Su ${ }^{1 *}$, Yibo Zhang ${ }^{5^{*}}$ and Hongxiong Guo ${ }^{6^{*}}$ (D)

\begin{abstract}
Background: To infer transmission direction of a HIV transmission chain is helpful not only in legal jurisdiction but also in precise intervention to prevent HIV spread. Recently, the direction of transmission is inferred by whether paraphyletic-monophyletic (PM) or a combination of paraphyletic and polyphyletic (PP) topologies is observed or not between the sequences of source and recipient in the phylogenetic tree. However, paraphyly between them often declines over time and may disappear between spouses due to bidirectional transmission after primary infection. In this study, our aim is to test the reliability of inferring HIV transmission direction between epidemiologically linked HIV-1 positive couples using whether or not paraphyly is observed in phylogenetic tree.

Methods: HIV quasi-species were sequenced using PCR product clones, and then Bayesian analysis of molecular sequences with MCMC was employed to construct phylogenetic relationship of env, gag, pol gene fragments of HIV-1 positive couples using BEAST software.

Results: Our results showed that all sequences of seven couples except pol sequences of couple 12 and 13 form their own monophyletic cluster in phylogenetic tree including the closest control sequences from GenBank or other studies on local samples, which are supported by significant Bayesian posterior probabilities more than 0.9932. Of seven couples, paraphyly is only observed in phylogenetic tree constructed with env and pol gene sequences of three couples and gag gene sequences of four couples. Paraphyly is not observed in half of HIV positive couples. Pol sequences of couple 13 is separated by Blast selected controls; pol sequences of couple 12 in phylogenetic tree is supported by a lower Bayesian posterior value.
\end{abstract}

(Continued on next page)

\footnotetext{
* Correspondence: sub602@126.com; yiboguan@126.com;

hongxiongguo@gmail.com

${ }^{1}$ Anhui Provincial Center for Disease Control and Prevention, 12560

Fanhuadadao, Hefei, China

${ }^{5}$ Department of Hospital Infection Control, Shanghai Ruijin Hospital Affiliated

to Shanghai Jiao Tong University School of Medicine, Shanghai 200025,

China

${ }^{6}$ Jiangsu Provincial Center for Disease Control and Prevention, 172 Jiangsu

Road, Nanjing, China

Full list of author information is available at the end of the article
}

(c) The Author(s). 2019 Open Access This article is distributed under the terms of the Creative Commons Attribution 4.0 International License (http://creativecommons.org/licenses/by/4.0/), which permits unrestricted use, distribution, and reproduction in any medium, provided you give appropriate credit to the original author(s) and the source, provide a link to the Creative Commons license, and indicate if changes were made. The Creative Commons Public Domain Dedication waiver (http://creativecommons.org/publicdomain/zero/1.0/) applies to the data made available in this article, unless otherwise stated. 


\begin{abstract}
(Continued from previous page)
Conclusion: Paraphyly relationship between sequences of donator and recipient is only observed among partial HIV-1 positive couples with epidemiological link. Phylogenetic relationship is not always the same when various gene regions of HIV are used to conduct phylogenetic analysis. The combination of phylogenetic analysis based on various gene regions of HIV and enough epidemiology investigation is essential when inferring transmission direction of HIV in a transmission chain or in one couple. However, while observed paraphyly can be used to infer transmission direction in HIV-1 positive couple, no observed paraphyly cannot deny it.
\end{abstract}

Keywords: HIV, Transmission direction, Quasi-species, Phylogenetic analysis, Source identification of HIV infection,

\section{Background}

Phylogenetic inference of microorganisms' transmission routes helps humans to understand epidemiologic dynamics of specific microorganism between various regions and species [1-4]. For those viruses with the ability of high diversity, phylogenetic reconstruction is often used to interpret transmission events. For example, phylogenetic analysis was used to not only identify the origin of high pathogenic influenza virus when high pathogenic influenza virus is present, but also to interpret HIV transmission route between various regions or provinces in a country $[5,6]$. HIV transmission among various individuals is often involved in legal disputes between donator and recipient $[7,8]$. In some cases, phylogenetic relation of HIV sequences from doubtful donator and recipient was central to the evidence of guilt [9]. Especially, to infer the transmission direction in a transmission chain is vital in these legal cases. Moreover, the interpretation of phylogenetic trees has a broader importance beyond criminal investigations, especially in public health investigations and practices.

Between two epidemiologically linked HIV-1 positive individuals, three possible ways of transmission exist. The first is direct transmission, the second is that there is one intermediary between two HIV-1 positive individuals, the last is that two HIV-1 positive individuals share a common infection source. To infer transmission direction between them, Yang et al. consecutively sampled in various time points for transmission pairs and identified transmission direction by observing the coreceptor switch of HIV from CCR5 to CXCR4 in vivo. Although the accuracy of this method, is up to $94.5 \%$, it takes a long time to identify the direction between transmission pairs, and is only applicable to those viruses using CCR5 coreceptor in the early phase of infection [10]. Therefore, the more common method is to reconstruct phylogenetic relationship using the samples collected at the same time point.

In phylogenetic tree, the paraphyly relationship exists between the donor's sequences and the recipient's $[11,12]$. Consequently, the transmission direction was inferred [13]. However, the paraphyly relationship between donor's sequences and the recipient's often decreases over time because evolution of HIV-1 in vivo in different bodies [11]. Moreover, the evolution of the various gene regions of HIV faces different pressure and experiences different models in vivo [13]. Which implies paraphyly relationship is not always observed, and paraphyly relationship is inconsistent between various gene regions of among epidemiologically linked HIV-1 positive pairs. In this study, we selected seven HIV-1 positive couples to explore the phylogenetic relationship of HIV among them and to analyze the feasibility of using observed paraphyly to infer transmission direction.

\section{Methods \\ Epidemiological data and samples \\ Design}

Firstly, to select HIV positive spouses, where only one party of HIV positive spouse has HIV risk behavior including sexual transmission and drug using, and the other party is infected with HIV through sexual transmission with her/his spouse. Secondly, to amplify the fragment of env, gag, pol gene, and to construct the clone of PCR amplificons. The various bacteria clone transinfected with PCR amplificons were sequenced to analyze HIV quasispecies in spouse. Finally, the paraphyletic relationship between HIV quasi-species sequences from spouse in MCMC phylogenetic tree was observed.

\section{Subjects}

Seven HIV-1 positive couples were investigated for their sexual behaviors which includes probable infectious route, the history of extramarital sexual behaviors, and history of intravenous drug injection. None knew their own and corresponding spouse's status of HIV infection before confirmed HIV infection.

\section{Samples}

Whole blood samples were collected using sterile ethylenediaminetetra-acetic acid tubes. The plasma was centrifugally separated at $3000 \mathrm{rpm}$ within $6 \mathrm{~h}$, then kept at $-80^{\circ} \mathrm{Cfor}$ viral RNA extraction. The study was reviewed and approved by the ethical committee at the Anhui Center for Disease Control and Prevention. 
Table 1 The basic information of seven couples

\begin{tabular}{|c|c|c|c|c|}
\hline Code & Sex & Diagnosis date & $\begin{array}{l}\text { The history of } \\
\text { extramarital } \\
\text { sex behavior }\end{array}$ & Ethnic group \\
\hline \multirow[t]{2}{*}{3} & Female & Feb 22,2011 & No & Han \\
\hline & male & Jan 27,2011 & Yes & Han \\
\hline \multirow[t]{2}{*}{7} & Female & Mar 20,2013 & Yes & Dai \\
\hline & male & Mar 20,2013 & Yes & Han \\
\hline \multirow[t]{2}{*}{8} & Female & Sep 18,2013 & No & Han \\
\hline & male & Sep 23,2013 & Yes & Han \\
\hline \multirow[t]{2}{*}{9} & Female & Feb 28,2013 & No & Han \\
\hline & male & $\operatorname{Jan} 22,2013$ & Yes & Han \\
\hline \multirow[t]{2}{*}{10} & Female & Nov 15,2013 & No & Han \\
\hline & male & Oct 24,2013 & Yes & Han \\
\hline \multirow[t]{2}{*}{12} & Female & $\operatorname{Jan} 2,2104$ & No & Han \\
\hline & male & Sep 1,2014 & $\begin{array}{l}\text { Intravenous } \\
\text { drug user }\end{array}$ & Han \\
\hline \multirow[t]{2}{*}{13} & Female & Dec31,2014 & No & Han \\
\hline & male & $\operatorname{Dec} 31,2014$ & Yes & Han \\
\hline
\end{tabular}

Written informed consent was obtained from all participants after we informed them of the objective of this study.

\section{RNA extraction, PCR, Clone, and sequencing}

Viral RNA was extracted from $140 \mu \mathrm{L}$ of plasma using QIAamp Viral RNA Mini kit (Qiagen, Valencia, CA). HIV-1 segments of env(C2-V3), gag (p17 and partial p24) and pol (protease and p51RT) were amplified using reverse transcriptase (RT)-nested polymerase chain reaction (PCR). The first PCR reactions were performed using the Superscript TM III one-step RT-PCR system with platinum Taq DNA polymerase (Invitrogen) with outer primer pair gp41-1 s/gp41-2as-B, GAG-L/GAGE2, and MAW26/RT21 to amplify env, gag, pol region of HIV-1, respectively.

The second PCR reactions were performed using the TaKaRa ExTaq kit (TaKaRa Biotechnology Co. Ltd., Dalian, China) with inner primer pair gp41-3 s/gp414as-xw, GAGF2/c-gag, and PRO-1/RT20 to amplify env, gag, and pol region of HIV-1, respectively. The sequences of primers used in this study have been previously described in detail $[14,15]$. Amplified PCR products were separated on an agarose gel and purified with QIAquick gel extraction kit (Qiagen, Valencia, CA). Purified products were cloned into pTV $118 \mathrm{~N}$ DNA plasmid, and transfected into E. Coli and cultured overnight, then selected 10 bacteria colonies to sequence DNA directly using an automated ABI 3730/3730xl DNA analyzer by Beijing Biomed BioTechnologies Co., Ltd.

\section{Phylogenetic analysis}

The clone sequences of every couple were used to search GenBank and aligned with the local sequences in other studies using BLAST program to identify the best matching HIV-1 RNA sequences. BLAST score significance was the criterion for selection. We rationalized that finding the highest matching HIV-1 sequences would increase the chances of refuting the priori hypothesis that couple's sequences form a monophyletic clade. The reference sequences included the best matching HIV-1 RNA sequences using BLAST program and the local control sequences. The local control sequences include all sequences that were gotten in previous

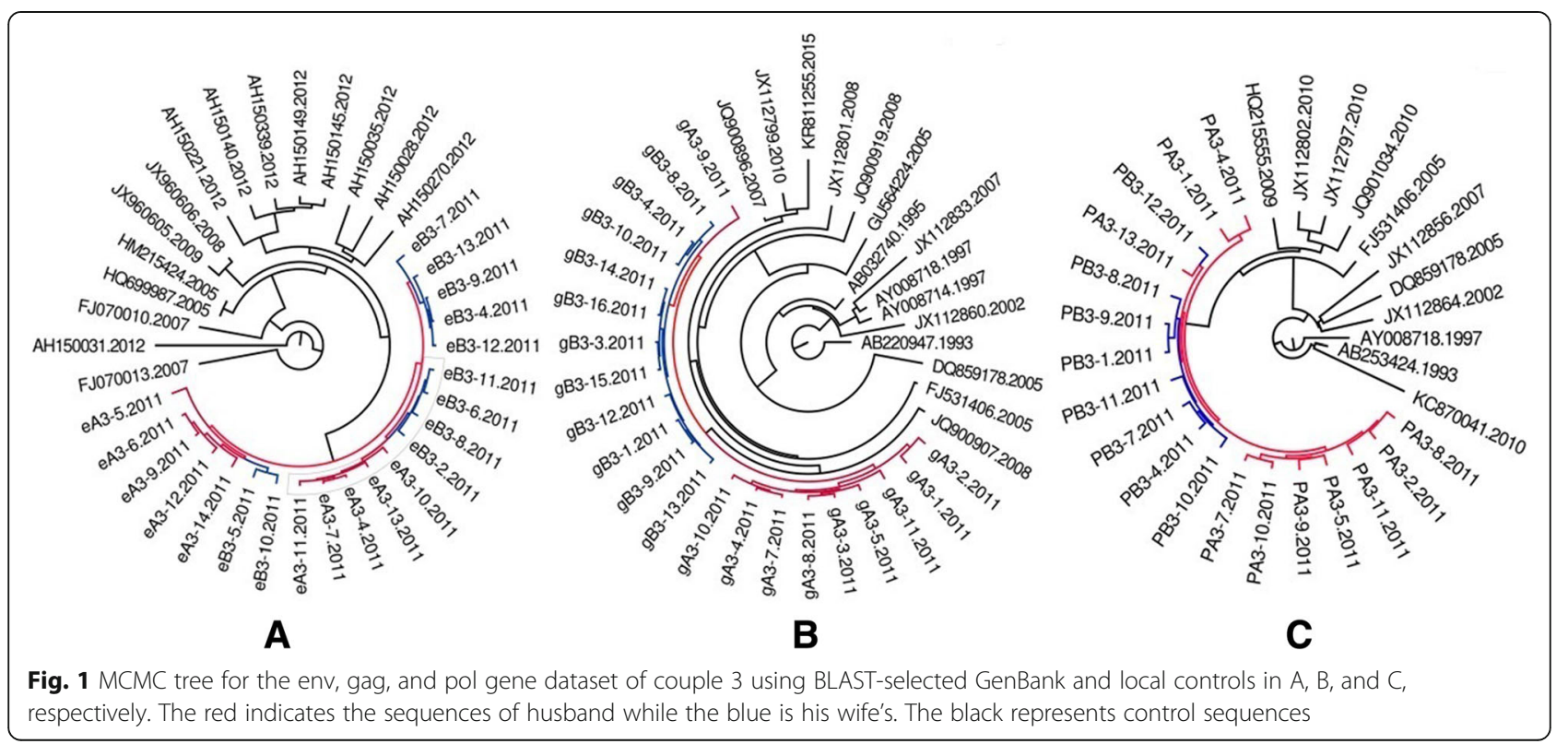




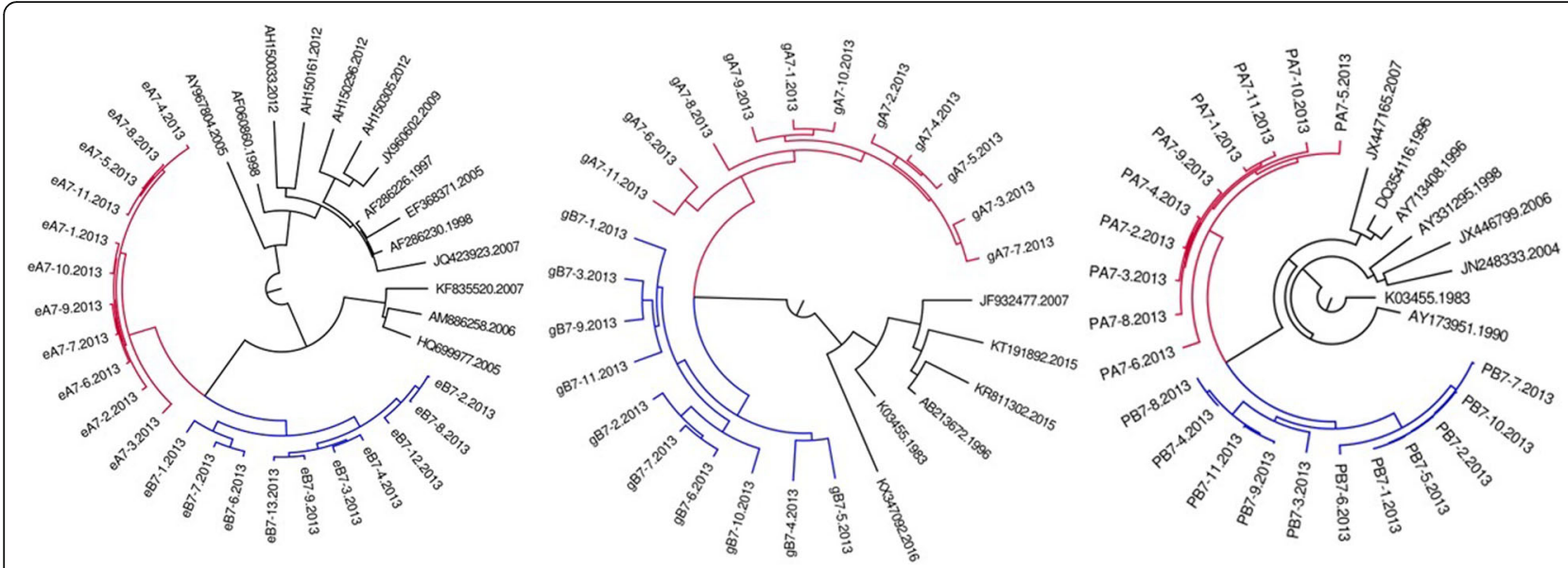

Fig. 2 MCMC tree for the env, gag, and pol gene dataset of couple 7 using BLAST-selected GenBank and local controls in A, B, and C, respectively. The red indicates the sequences of husband while the blue is his wife's. The black represents control sequences

studies, molecular epidemiology investigation and drug resistance surveillance. Nucleotide sequences were aligned with reference strains using the Clustal X2, and phylogenetic analyses were performed in BEAST v.1.82. To assess the appropriate model of evolution for the phylogenetic analysis of the env, gag, pol gene datasets, likelihood ratio tests were conducted using jModelTest software. Both the general time-reversal (GTR) and Hasegawa-Kishino-Yano nucleotide substitution models with a gamma distribution model of among site rate heterogeneity were employed. The Markov chain Monte Carlo (MCMC) search was run for $5 \times 10^{6}$ generations with trees sampled every 100th generation. Burn-in was set at $20 \%$ and a posterior consensus tree generated from 50,000 trees sampled. The MCMC output was tested for convergence and effective sample size using Tracer v1.4.

\section{Result}

Subjects' basic characteristics

Of all men among seven HIV-1 positive couples, five males of them had the experience of extramarital sexual behavior including commercial sex activity; one was an intravenous drug user. Only one female had extramarital sexual behavior and is a Dai nationality (shown in Table 1). Among them, only one side of every couple had the risk behavior causing HIV infection before marriage. Six of these couples, husband and wife were confirmed HIV infection within 1 month, and only one within 8 months.

\section{Phylogenetic relationship of various gene region of HIV-1 positive couples}

All HIV-1 sequences of couple 3,7,8,9, and 10 clusters a monophyly clade with a well support of Bayesian

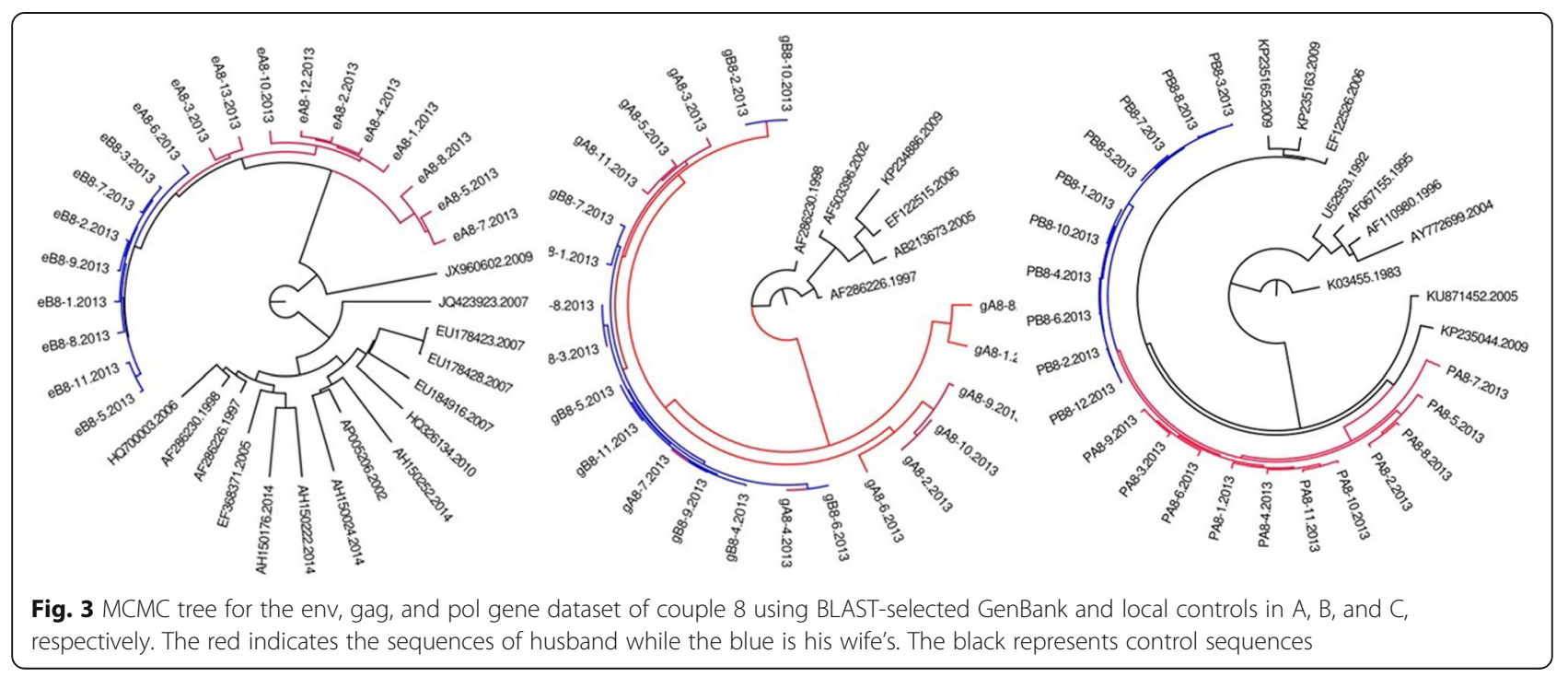




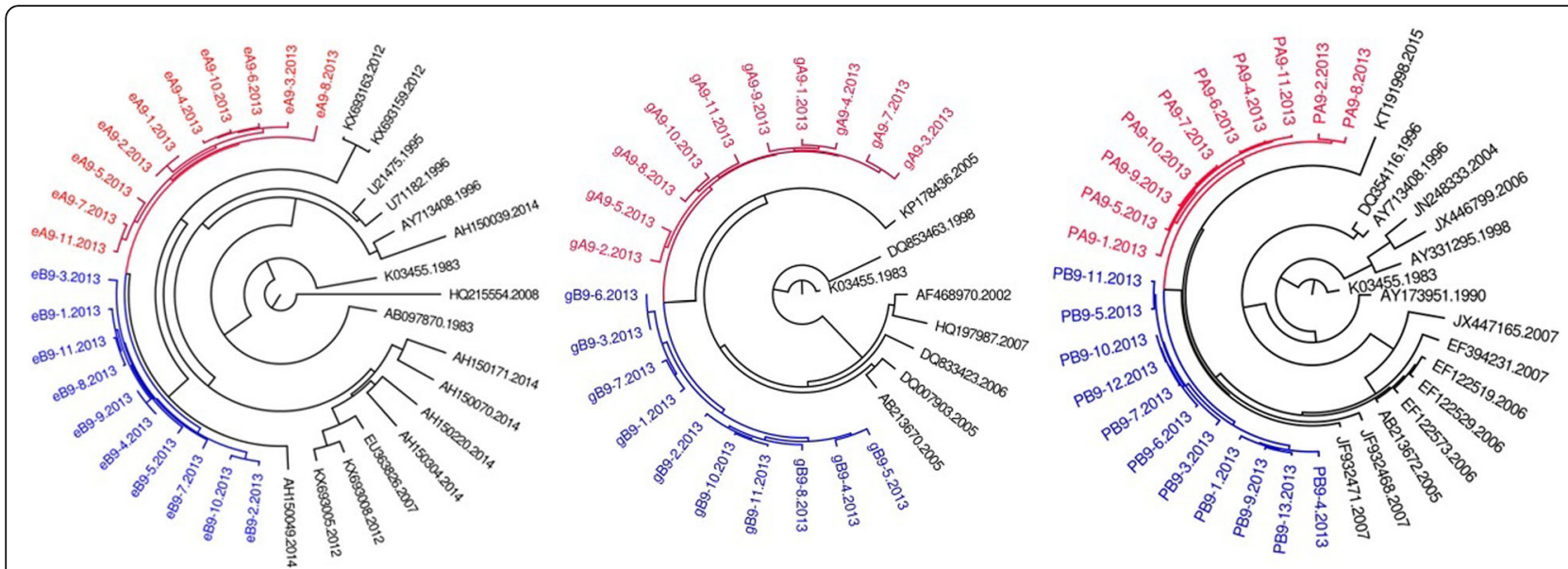

Fig. 4 MCMC tree for the env, gag, and pol gene dataset of couple 9 using BLAST-selected GenBank and local controls in A, B, and C, respectively. The red indicates the sequences of husband while the blue is his wife's. The black represents control sequences

posterior probability (as showed in Figs. 1, 2, 3, 4, and 5), respectively. As of couple 12 and 13, the sequences of env and gag formed a monophyly clade (Figs. 6a, b, 7a, and $b$ ) while the sequences of pol of couple 12 and 13 are separated by blast selected controls (Figs. 6c, and 7c).

As summarized in Table 2, among six of seven HIV-1 positive couples, the phylogenetic relationship between HIV sequences of couple is in accordance with in env, gag pol. Only couple 13, the phylogenetic relationship in gag region is not accordance with that of env and pol. Of them, three are PM, three are MM, one is PM in gag while MM in env and pol.

\section{Discussion}

In this study, seven epidemiologically linked HIV-1 positive couples were used to infer phylogenetic relation based on quasi-species sequences of HIV-1. Epidemiology investigation confirms that only one side of every couple experienced high-risk behavior associated with HIV-1 infection. The other side of every couple has the unique chance to get HIV infection from his/her spouse. Phylogenetic analysis also shows that all sequences of env and gag region of HIV-1 form a monophyly with respect to controls, which supports that HIV-1 infected each couple shares with the most recent common ancestor and is accordance with the results of epidemiological investigation.

In previous studies, source in a transmission chain was inferred based on observed paraphyly between source and recipient with respect to controls [13]. In our study, paraphyly between source and recipient was only observed in three of seven couples amongst env, gag and pol phylogenetic trees. In couple 13, paraphyly was only observed in gag phylogenetic tree. The rest of them are monophyletic, using the sequences of env, gag or pol of HIV-1. Our findings

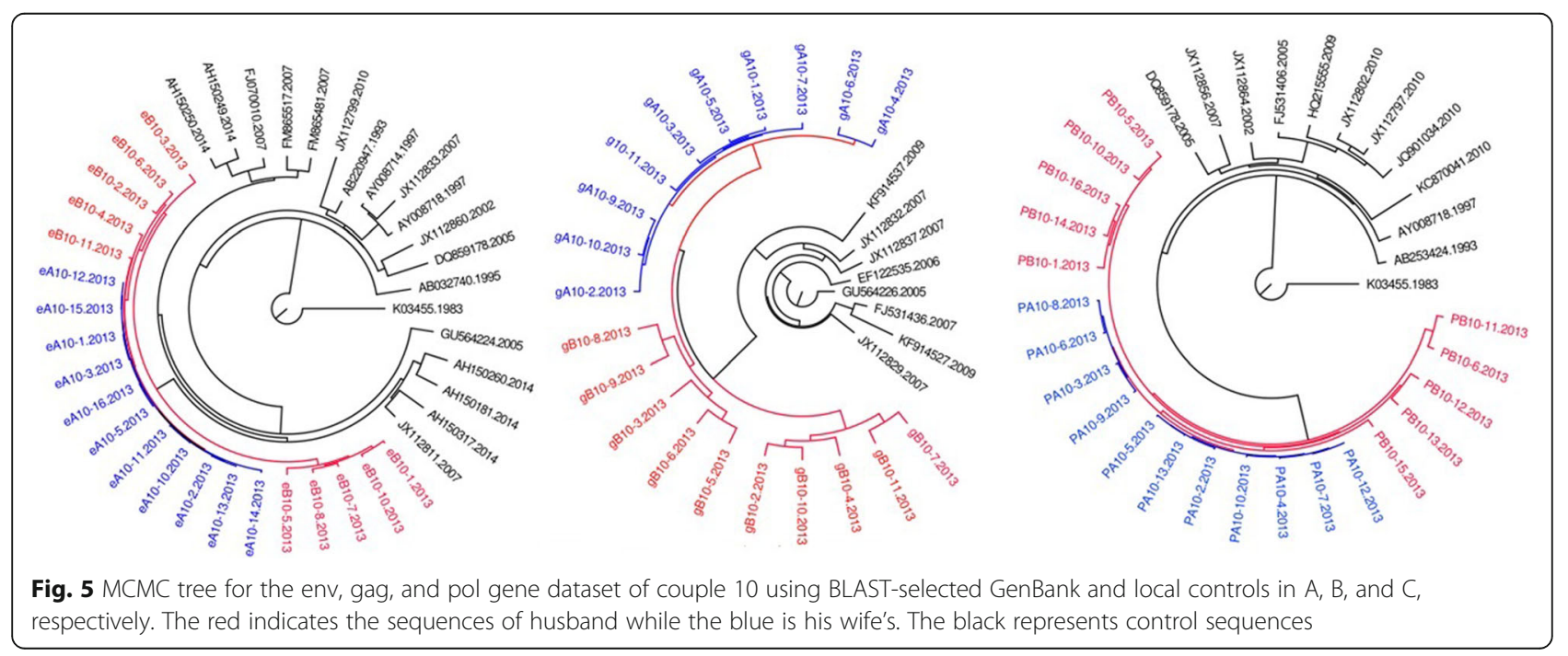




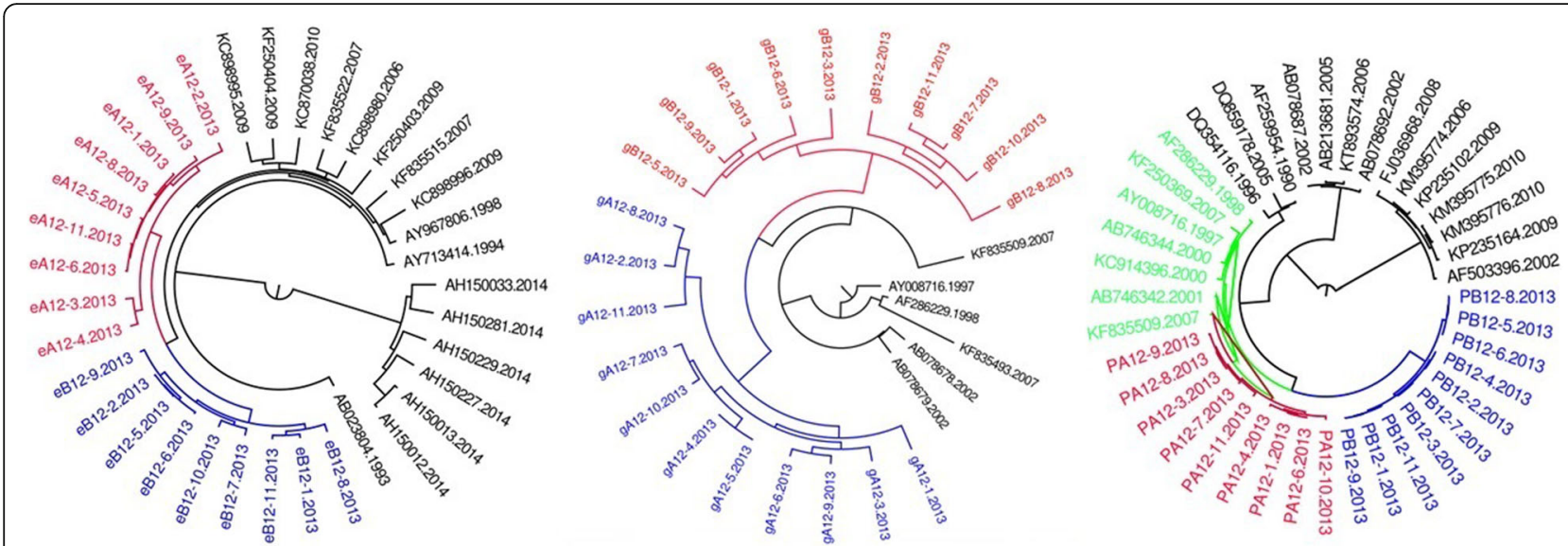

Fig. 6 MCMC tree for the env, gag, and pol gene dataset of couple 12 using BLAST-selected GenBank and local controls in A, B, and C, respectively. The red indicates the sequences of husband while the blue is his wife's. The black and emerald represents control sequences

indicate that paraphyly is not always observed between source and recipient. As we knew, the survival of HIV-1 in vivo experienced pressures from many aspects such as host's immune system, antiretroviral therapy, which will lead to the loss of HIV diversity in vivo [16-21]. Therefore, paraphyly of source sequences with respect to recipient sequences will decline over time. It suggested that source can be inferred based on the observed paraphyly, and not be denied when not observing paraphyly. Our finding is consistent with the results by Diane et al [13]. In addition, recombination among viral sequences within the source individual will degrade support for particular paraphyletic relationships over time. In this study, we did not observe paraphyly between source and recipient among less than half of HIV-1 positive couples. As of HIV-1 positive couple living together for long term, HIV-1 transmission should be directional.
In this occasion, it become more difficult to infer transmission direction using phylogenetic method. Moreover, it implies that half of persons were untested during the window periods of acute infection.

For most of HIV-1 couples, the phylogenetic relationship on env, gag, pol is consistent (couple 3, 8, 9), and with a support of higher priority value. However, there is always an exception. For example, although the pol sequences of couple 12 form a monophyly with respect to controls, the value of Bayesian posterior probability is very low (only 0.6431 ). Moreover, paraphyly is observed in phylogenetic tree based on gag region sequences of couple 13 while not appearing in that based on env and pol. Furthermore, the pol sequences of couple 13 are separated by Blast selected controls. The selection pressures that various gene region of HIV face in vivo are different. As we knew, the selective pressure which HIV faces in vivo is different between various gene regions.

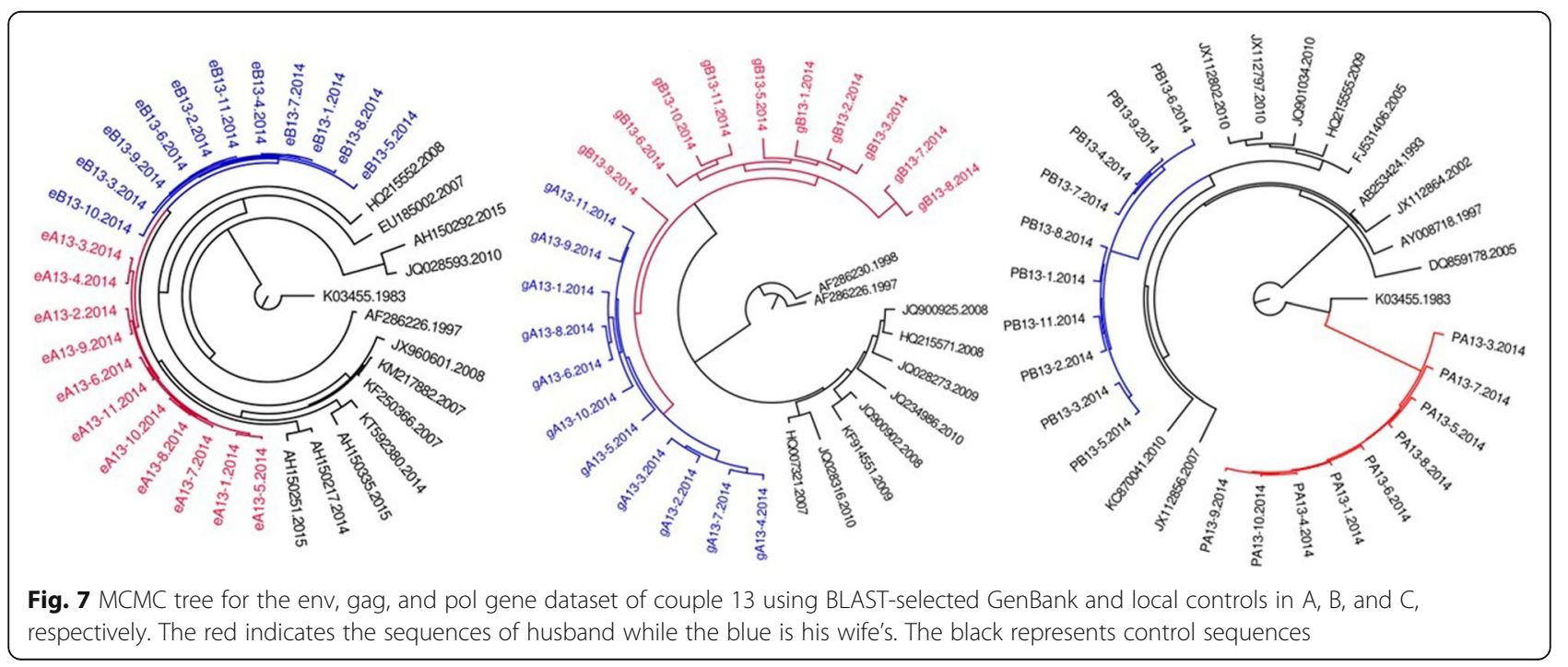


Table 2 The phylogenetic relationship between HIV-1 positive couples

\begin{tabular}{|c|c|c|c|c|c|c|}
\hline \multirow[t]{3}{*}{ Code } & \multicolumn{6}{|l|}{ Gene Region } \\
\hline & \multicolumn{2}{|l|}{ env } & \multicolumn{2}{|l|}{ gag } & \multicolumn{2}{|l|}{ pol } \\
\hline & $\begin{array}{l}\text { Phylogenetic } \\
\text { relationship }\end{array}$ & $\begin{array}{l}\text { Bayesian posterior } \\
\text { probability }\end{array}$ & $\begin{array}{l}\text { Phylogenetic } \\
\text { relationship }\end{array}$ & $\begin{array}{l}\text { Bayesian posterior } \\
\text { probability }\end{array}$ & $\begin{array}{l}\text { Phylogenetic } \\
\text { relationship }\end{array}$ & $\begin{array}{l}\text { Bayesian posterior } \\
\text { probability }\end{array}$ \\
\hline 3 & PM & 0.9998 & PM & 0.9986 & PM & 0.9999 \\
\hline 7 & MM & 0.9998 & MM & 0.9932 & MM & 0.9932 \\
\hline 8 & PM & 0.9997 & PM & 0.9998 & PM & 0.9978 \\
\hline 9 & MM & 0.9997 & MM & 0.9997 & MM & 0.9971 \\
\hline 10 & PM & 0.9997 & PM & 0.999 & PM & 0.9981 \\
\hline 12 & MM & 0.9997 & MM & 0.9941 & $\mathrm{MM}$ & 0.6431 \\
\hline 13 & MM & 0.9997 & PM & 0.9997 & MM & - \\
\hline
\end{tabular}

The most selective pressure was observed in env region, followed by pol region. Gag region is relatively conservative. Therefore, it is necessary to conduct phylogenetic analysis on various gene regions of HIV at the same time during inferring transmission direction.

\section{Conclusions}

Paraphyly relationship between sequences of donator and recipient is a vital indication to infer transmission direction in HIV transmission chain. However, it is not applicable to all HIV transmission chains due to loss of paraphyly after infection for some time, especially for those spouse or sex partners living together, and intravenous drug users sharing common syringe over a long term [22]. Moreover, phylogenetic relationship is not always same when various gene regions of HIV are used to conduct phylogenetic analysis. Therefore, the combination of phylogenetic analysis based on various gene regions of HIV and enough epidemiology investigation is essential when inferring transmission direction of HIV in a transmission chain or only a couple.

\section{Abbreviations}

BEAST: Bayesian evolutionary analysis sampling tree; GTR: General timereversal; HCV: Hepatitis C virus; HIV: Human immunodeficiency virus; HIV1: Human immunodeficiency virus type one; MCMC: Markov chain Monte Carlo; MEGA: Molecular evolutionary genetics analysis

\section{Acknowledgements}

We thank the staff of Hefei prefecture Center for Disease Control and Prevention for their work in specimens' collection and epidemiology investigation.

\section{Authors' contributions}

JW, PZ, YF, HX, BS, YZ, HG conceived this work. JW, YZ and HG performed phylogenetic analysis and edit the manuscript. ZH, HY, HW, YL conducted epidemiology investigate and collected samples. YS, LJ, AL, YQ, LM performed experiment. All authors read and approved the final manuscript.

\section{Funding}

This project was supported partly by the project funded by Anhui provincial Program of Prevent medicine \& Public Health (Grant\#2017jk002), National Grand Program on Key Infectious Disease Control (Grant\#2017ZX10201101), Jiangsu provincial High-Level Talents Programs in Health (LGY2016021), and Gaoyuan Clinical Medicine Grant Support (No. 2017269). The funder had no role in the study design, data collection and analysis, decision to publish, or preparation of the manuscript.

\section{Availability of data and materials}

The datasets used in this study are available from the corresponding author on reasonable request.

Ethics approval and consent to participate

This study was approved by the Medical Ethics Committee of Anhui Provincial Center for Disease Control and Prevention. All subjects signed informed consent form before collecting samples and epidemiology investigation.

\section{Consent for publication}

Not applicable.

\section{Competing interests}

The authors declare that they have no competing interests.

\section{Author details}

${ }^{1}$ Anhui Provincial Center for Disease Control and Prevention, 12560 Fanhuadadao, Hefei, China. ${ }^{2}$ Hefei Prefecture Center for Disease Control and Prevention, 86 Liu'an Road, Hefei, China. ${ }^{3}$ Shanghai Municipal Center for Disease Control and Prevention, 1380 Zhongshan West Road, Shanghai, China. ${ }^{4}$ Chinese Center for Disease Control and Prevention, 155 Changbei Road, Beijing, China. ${ }^{5}$ Department of Hospital Infection Control, Shanghai Ruijin Hospital Affiliated to Shanghai Jiao Tong University School of Medicine, Shanghai 200025, China. ${ }^{6}$ Jiangsu Provincial Center for Disease Control and Prevention, 172 Jiangsu Road, Nanjing, China.

Received: 17 September 2018 Accepted: 4 June 2019 Published online: 28 June 2019

\section{References}

1. Lu L, Tong W, Gu L, Li C, Lu T, Tee KK, Chen G. The current hepatitis C virus prevalence in China may have resulted mainly from an officially encouraged plasma campaign in the 1990s: a coalescence inference with genetic sequences. J Virol. 2013;87(22):12041-50.

2. Chen H, Deng Q, Ng SH, Lee RTC, Maurer-Stroh S, Zhai W. Dynamic convergent evolution drives the passage adaptation across 48 years'history of H3N2 influenza evolution. Mol Biol Evol. 2016;33(12):3133-43.

3. Qi W, Jia W, Liu D, Li J, Bi Y, Xie S, Li B, Hu T, Du Y, Xing L, et al. Emergence and adaptation of a novel highly pathogenic H7N9 influenza virus in birds and humans from a 2013 human-infecting low-pathogenic ancestor. J Virol. 2018;92(2):e00921-17.

4. Gao F, Bailes E, Robertson DL, Chen Y, Rodenburg CM, Michael SF, Cumminsk LB, Arthur LO, Peeters M, Shaw GM, et al. Origin of HIV-1 inthechimpanzee Pantroglodytes troglodytes. Nature. 1999:397:436-41.

5. Plantier JC, Leoz M, Dickerson JE, De Oliveira F, Cordonnier F, Lemee V, Damond F, Robertson DL, Simon F. A new human immunodeficiency virus derived from gorillas. Nat Med. 2009;15(8):871-2. 
6. Mumtaz G, Hilmi N, Akala FA, Semini I, Riedner G, Wilson D, Abu-Raddad LJ. HIV-1 molecular epidemiology evidence and transmission patterns in the Middle East and North Africa. Sex Transm Infect. 2011;87(2):101-6.

7. Siljic M, Salemovic D, Cirkovic V, Pesic-Pavlovic I, Ranin J, Todorovic M, Nikolic S, Jevtovic D, Stanojevic M. Forensic application of phylogenetic analyses - exploration of suspected HIV-1 transmission case. Forensic Sci Int Genet. 2017;27:100-5.

8. Metzker ML, Mindell DP, Liu XM, Ptak RG, Gibbs RA, Hillis DM. Molecular evidence of HIV-1 transmission in a criminal case. Proc Natl Acad Sci U S A. 2002;99(22):14292-7.

9. Oliveira TD, Pybus OG, Rambaut A, Salemi M, Cassol S, Ciccozzi M, Rezza G, Gattinara CG, D'Arrigo R, Amicosante M, et al. HIV-1 and HCV sequences from Libyan outbreak. Nature. 2006:444:836-7.

10. Yang J, Ge M, Pan X-M. A time lag insensitive approach for estimating HIV-1 transmission direction. AIDS. 2012;26:921-8.

11. Romero-Severson EO, Bulla I, Leitner T. Phylogenetically resolving epidemiologic linkage. PNAS. 2016;113(10):2690-5.

12. Thomas L, Escanilla D, Franzen C, Uhlen M, Albert J. Accurate reconstruction of a known HIV-1 transmission history by phylogenetic tree analysis. PNAS. 1996;93(20):10864-9.

13. Scaduto DI, Brown JM, Haaland WC, ZwickI DJ, Hillis DM, Metzker ML. Source identification in two criminal cases using phylogenetic analysis of HIV-1 DNA sequences. PNAS. 2010;107(50):21242-7.

14. Wu J, Shen Y, Zhong P, Feng Y, Xing H, Jin L, Qin Y, Liu A, Miao L, Cui L, et al. The predominant cluster of CRF01_AE circulating among newly diagnosed HIV-1-positive people in Anhui Province, China. AIDS Res Hum Retrovir. 2015;31(9):926-31.

15. Guo H, Hu H, Zhou Y, Yang H, Huan X, Qiu T, Fu G, Ding P. A novel HIV-1 CRF01_AE/B recombinant among men who have sex with men in Jiangsu Province, China. AIDS Res Hum Retrovir. 2014;30(7):706-10.

16. Chiu Y-L, Soros VB, Kreisberg JF, Stopak K, Yonemoto W, Greene WC. Cellular APOBEC3G restricts HIV-1 infection in resting CD41 T cells. Nature. 2005; 345(7038):108-14.

17. Luban J, Soll SJ, Wilson SJ, Kutluay SB, Hatziioannou T, Bieniasz PD. Assisted evolution enables HIV-1 to overcome a high TRIM5a-imposed genetic barrier to rhesus macaque tropism. PLoS Pathog. 2013;9(9):e1003667.

18. de Azevedo SSD, Caetano DG, Côrtes FH, Teixeira SLM, dos Santos Silva K, Hoagland B, Grinsztejn B, Veloso VG, Morgado MG, Bello G. Highly divergent patterns of genetic diversity and evolution in proviral quasispecies from HIV controllers. Retrovirology. 2017;14(1):29.

19. Maldarelli F, Kearney M, Palmer S, Stephens R, Mican J, Polis MA, Davey RT, Kovacs J, Shao W, Rock-Kress D, et al. HIV populations are large and accumulate high genetic diversity in a nonlinear fashion. J Virol. 2013;87(18): 10313-23.

20. Santa-Marta M, de Brito PM, Godinho-Santos A, Goncalves J. Host factors and HIV-1 replication: clinical evidence and potential therapeutic approaches. Front Immunol. 2013;4.

21. Pernas MA, Casado CN, Arcones C, Llano A, Sánchez-Merino VC, Mothe B, Vicario JL, Grau E, Ruiz L, Sánchez J, et al. Low-replicating viruses and strong anti-viral immune response associated with prolonged disease control in a superinfected HIV-1 LTNP elite controller. PLoS One. 2012;7(2):e31928.

22. Abecasis $A B$, Pingarilho $M$, Vandamme $A M$. Phylogenetic analysis as a forensic tool in HIV transmission investigations. AIDS. 2018;32(5):543-54.

\section{Publisher's Note}

Springer Nature remains neutral with regard to jurisdictional claims in published maps and institutional affiliations.

Ready to submit your research? Choose BMC and benefit from:
- fast, convenient online submission
- thorough peer review by experienced researchers in your field
- rapid publication on acceptance
- support for research data, including large and complex data types
- gold Open Access which fosters wider collaboration and increased citations
- maximum visibility for your research: over 100M website views per year
At BMC, research is always in progress.
Learn more biomedcentral.com/submissions

